\title{
Reabilitação vascular não supervisionada em indivíduos com doença arterial periférica
}

\author{
Non-supervisioned vascular rehabilitation in individuals with peripheral arterial disease
}

\author{
Carolina Machado de Melo Felix (1), Débora Pantuso Monteiro (1, \\ Danielle Aparecida Gomes Pereira (D) ${ }^{1} \bowtie$ \\ ${ }^{1}$ Universidade Federal de Minas Gerais (UFMG), Belo Horizonte, MG, Brasil.
}

RESUMO

Introdução: A reabilitação não supervisionada para indivíduos com doença arterial periférica (DAP) tem como objetivo incentivar os pacientes a praticar exercício físico, imprescindível para melhora do sintoma claudicante e da capacidade funcional.

Objetivo: Avaliar os resultados do exercício não supervisionado em indivíduos com DAP no que se refere à capacidade funcional, resistência muscular e autopercepção de funcionalidade.

Materiais e Métodos: Trata-se de um estudo retrospectivo que avaliou dados de prontuários de 11 indivíduos com DAP que realizaram reabilitação vascular não supervisionada à distância, durante dois meses, com orientações e reavaliações mensais. A reabilitação foi composta por orientações sobre como o indivíduo deveria realizar o exercício, diário de registro para controle da realização dos mesmos e escala de Borg modificada para quantificar a intensidade. Para as avaliações foram utilizados o Heel-Rise Test (HRT), o Incremental Shuttle Walk Test (ISWT) e o Walking Impairment Questionnaire (WIQ).

Resultados: Uma melhora significativa foi observada no primeiro mês de atividade física não supervisionada em

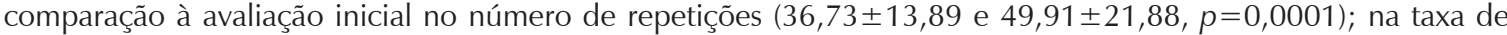
repetição no $\operatorname{HRT}(0,68 \pm 017$ repetições/segundo e $0,84 \pm 020$ repetições/segundo, $p=0,004)$; na distância de caminhada $(207,27 \pm 97,68$ metros e $266,36 \pm 113,87$ metros, $p=0,0001)$; na economia de caminhada no ISWT $(2,07 \pm 1,02$ metros/frequência cardíaca e 2,59 $\pm 1,08$ metros/frequência cardíaca, $p=0,001)$; na graduação da dor $(15,91 \pm 12,61 \%$ e $43,18 \pm 25,23 \%, p=0,0001)$ e velocidade de caminhada $(21,84 \pm 26,91 \%$ e $38,59 \pm 27,49 \%$, $p=0,017)$ do questionário WIQ.

Conclusão: $O$ treinamento não supervisionado, com orientações sistematizadas, é uma opção terapêutica segura e apresenta resultados satisfatórios na capacidade funcional, resistência muscular e autopercepção de funcionalidade.

Palavras-chave: doença arterial periférica; fisioterapia; autocuidado.

ABSTRACT

Introduction: The non-supervised rehabilitation for individuals with peripheral artery disease (PAD) has as objective to motivate patients to practice physical exercise, fundamental for the improvement of the claudicating symptom and functional capacity.

Objective: To evaluate the results of a non-supervised exercise in individuals with PAD regarding functional capacity, muscle resistance, and self-perception of functionality.

Materials and Methods: This is a retrospective study, which evaluated data from medical records of 11 individuals with PAD who underwent remote unsupervised vascular rehabilitation for two months, with monthly guidelines and reevaluations. The rehabilitation was composed by guidelines on how the individual should perform the exercise, log journal to control their performance and modified Borg scale to quantify the intensity. For the evaluations the Heel-Rise test (HRT), Incremental Shuttle Walk Test (ISWT), and the Walking Impairment Questionnaire (WIQ) were used.

Results: A statistically significant improvement was observed in the first month of non-supervised physical activity in comparison to the initial evaluation, regarding the number of repetitions (36.73 \pm 13.89 repetitions and $49.91 \pm 21.88$ repetitions, $p=0.0001$ ), $H R T$ repetition rate $(0.68 \pm 017$ repetitions/second and $0.84 \pm 020$ repetitions/second, $p=0.004)$, the walk distance $(207.27 \pm 97.68$ meters and 266.36 \pm 113.87 meters, $p=0.0001)$, ISWT walk economy $(2.07 \pm 1.02$ meters/heart rate, and $2.59 \pm 1.08$ meters/heart rate, $p=0.001)$ and in the WIQ pain graduation $(15.91 \pm 12.61 \%$ and $43.18 \pm 25.23 \%, p=0.0001)$ and walk speed $(21.84 \pm 26.91 \%$ and $38.59 \pm 27.49 \%, p=0.017$ ).

Conclusion: The non-supervised training, with systematic orientations, is a safe therapeutic option, and presents satisfactory results on functional capacity, muscle resistance, and self-perception of functionality.

Keywords: peripheral arterial disease; physical therapy specialty; self-care.

$\triangle$ Correspondência:

DANIELle APARECIDA GOMES Pereira

Universidade Federal de Minas Gerais

Av. Pres. Antônio Carlos, 6627 - Campus Pampulha

31270-901, Belo Horizonte, MG, Brasil

E-mail: danielleufmg@gmail.com 


\section{INTRODUÇÃO}

A doença arterial periférica (DAP) é caracterizada pela alteração na estrutura e função arterial devido a uma obstrução secundária à aterosclerose, acometendo comumente os membros inferiores ${ }^{1-3}$. A diminuição do fluxo sanguíneo leva ao aparecimento da claudicação intermitente ${ }^{3,4}$, definida como dor ou desconforto em um grupo muscular específico, induzida pelo exercício e aliviada com o repouso ${ }^{5-8}$. A claudicação intermitente está associada com um risco aumentado de morbidade cardiovascular e mortalidade ${ }^{9,10}$. Além disso, é o primeiro sintoma e o mais comum associado à DAP e resulta em limitação física, sendo a limitação na caminhada a principal queixa, diminuição da qualidade de vida além do prejuízo de aspectos sociais e emocionais ${ }^{2}$.

Programas de exercícios somados à modificação de fatores de risco possibilitam um melhor manejo das repercussões da doença ${ }^{6}$. Alguns dos efeitos do exercício são o aumento mínimo do fluxo colateral, melhora da vasodilatação óxido nítrico dependente, melhora da função mitocondrial, diminuição da inflamação sistêmica e, como consequência, redução dos sintomas, melhora da capacidade funcional e diminuição da ocorrência de eventos cardiovasculares levando a um melhor curso clínico da doença ${ }^{6,11}$. A atividade física aeróbia do tipo caminhada é a mais utilizada como tratamento para a DAP e é uma intervenção segura, eficaz e de baixo custo quando comparada com a intervenção cirúrgica ou uso de medicamentos ${ }^{12}$.

A prescrição de um programa de exercícios para indivíduos com DAP requer acompanhamento individualizado e direcionado para a educação do paciente, incluindo a modificação dos fatores de risco. Os programas podem ser supervisionados, não supervisionados, na instituição ou em domicílio e podem ser combinados com outras intervenções. Todas as estratégias de reabilitação dependem da adesão do paciente, porém, programas de exercício supervisionado vêm demonstrando resultados superiores aos programas não supervisionados realizados em casa ${ }^{6}$.

A reabilitação não supervisionada tem como objetivo principal exercitar pacientes sob supervisão indireta e pode ser uma alternativa viável e segura para abordagem de indivíduos com DAP e promover manutenção de benefícios obtidos no treinamento supervisionado. Tendo em vista a escassez de centros estruturados de reabilitação vascular no Brasil, deve ser considerada a possibilidade da existência de programas de exercícios não supervisionados aplicados de forma individualizada, com demonstrações práticas e acompanhamento sistematizado ${ }^{13,14}$. Além de gerar menos custos para o sistema de saúde, beneficia os indivíduos que não podem participar do serviço de reabilitação por dificuldades de transporte, dificuldades financeiras e sociais, impedindo a ampla utilização $0^{9,13,14}$.

Assim, objetivo do presente estudo foi avaliar os resultados do exercício não supervisionado em indivíduos com DAP no que se refere à capacidade funcional, resistência muscular e autopercepção de funcionalidade durante dois meses de acompanhamento em um projeto de extensão universitária.

\section{MATERIAIS E MÉTODOS}

Estudo exploratório retrospectivo de análise de prontuários de indivíduos com DAP atendidos em um ambulatório de reabilitação vascular que realizavam reabilitação não supervisionada com orientações sistematizadas com acompanhamento mensal no ambulatório. A coleta de dados das avaliações e reavaliações foi feita nos prontuários durante o período de março de 2016 a março de 2017.

Os critérios de inclusão foram: prontuários de indivíduos com diagnóstico de DAP encaminhados ao serviço de reabilitação vascular de um hospital universitário que realizaram reabilitação vascular não supervisionada. Os critérios de exclusão foram prontuários de indivíduos que não apresentavam pelo menos uma avaliação inicial e uma avaliação mensal registradas.

No ambulatório de reabilitação vascular é realizado o acompanhamento fisioterapêutico mensal de pacientes com dificuldade de acesso ao serviço, dificuldades de transporte, dificuldades financeiras ou sociais. É realizada uma avaliação inicial e avaliações a cada mês de exercício não supervisionado, sendo essas avaliações compostas de: avaliação fisioterapêutica global com aferição de dados vitais, o Heel-Rise Test (HRT), aplicação do Walking Impairment Questionnaire (WIQ) e a realização do Incremental Shuttle Walking Test (ISWT), nesta ordem. Para a realização do teste subsequente os dados vitais devem retornar aos valores basais. Todos os acompanhamentos do treinamento não supervisionado foram feitos por um único fisioterapeuta com treinamento prévio para a realização dos testes. Antes, durante e após as avaliações os participantes foram monitorados em relação à pressão arterial com esfigmomanomêtro $\left(\operatorname{Tycos}^{\circledR}\right)$, estetoscópio (Littman ${ }^{\circledR}$, Cardiology IV ${ }^{\mathrm{TM}}$ ) e a frequência cardíaca por meio do cardiofrequencímetro (Polar $\left.{ }^{\circledR}, \mathrm{FII}\right)$.

O HRT é um instrumento reprodutível e viável na prática clínica para a avaliação da função do músculo tríceps sural em indivíduos com DAP ${ }^{15}$. O teste foi realizado conforme previamente descrito na literatura ${ }^{15,16}$ e consistiu em realizar o número máximo de flexões plantares na amplitude máxima até o ponto de fadiga voluntária, na velocidade mais rápida possível. O examinador registrava o número de repetições realizadas e a quantidade total de tempo em segundos 
necessário para a realização do teste. A partir dessas duas variáveis é calculada a taxa de repetição (repetições/ segundo) $)^{15,16}$ (Figura 1).

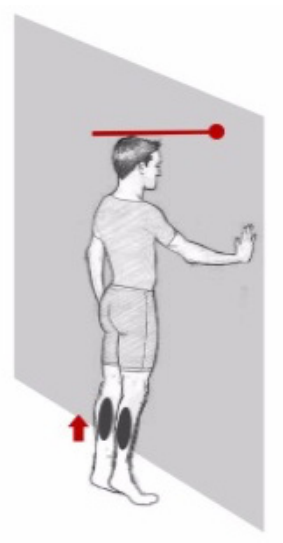

Figura 1. Heel-Rise Test.

O ISWT é um teste de caminhada incremental e progressivo, composto de 12 estágios, com confiabilidade testada em pacientes com DAP e apresenta como finalidade avaliar a capacidade funcional a partir da distância percorrida, sendo realizado conforme descrito na literatura' ${ }^{17,18}$. É um teste utilizado para avaliar resultados de intervenções e prognósticos, apresentando-se como um instrumento válido, confiável e seguro para a avaliar da capacidade funcional em indivíduos com doenças vasculares ${ }^{17}$.

A economia de caminhada é caracterizada pela absorção de oxigênio durante a deambulação. Pacientes com claudicação intermitente têm pouca economia de caminhada, pois sua absorção de oxigênio durante a deambulação é maior do que pacientes com ausência de DAP. A alta absorção de oxigênio que resulta em economia de caminhada prejudicada e o consumo máximo de oxigênio diminuído $\left(\mathrm{VO}_{2}\right.$ máximo) em pacientes com claudicação intermitente faz com que a caminhada livre de dor seja realizada com um alto custo metabólico, reduzindo a tolerância ao exercício. Após o início da dor há um aumento na absorção de oxigênio, além de um recrutamento de unidades motoras de contração rápida, o que aumentaria ainda mais a absorção de oxigênio durante o exercício, reduzindo assim a tolerância para sustentar a deambulação ${ }^{5,19}$. A economia de caminhada é um dos mecanismos para melhorar a distância de caminhada sem claudicação após treinamento com exercícios. O aumento no desempenho da caminhada representa uma melhora da capacidade metabólica ou uma mudança na economia de caminhada induzida pelo treinamento $0^{5,20}$. É expressa nesse estudo pela distância percorrida no ISWT dividida pela frequência cardíaca final do teste, representando quantos metros por batimento o paciente foi capaz de caminhar.

O WIQ é um questionário validado e reprodutível para a população brasileira com claudicação intermitente, utilizado para obtenção de informações sobre a autopercepção da capacidade de locomoção ${ }^{21,22}$. Esse questionário aborda aspectos referentes ao último mês e é composto por domínios: dor, distância, velocidade e subir escada ${ }^{21-23}$.

As variáveis analisadas no presente estudo foram o número de flexões plantares e a taxa de repetição (flexões plantares por segundo) no HRT; a distância total e a economia de caminhada no ISWT e a pontuação percentual em cada domínio no WIQ.

Após a avaliação os pacientes recebiam orientações individualizadas sobre os exercícios que deveriam ser realizados, bem como orientações para evitar fatores de risco que envolvem a DAP. Um dos exercícios orientados para o domicílio era a caminhada até próximo da dor máxima com duração de no mínimo trinta minutos desconsiderando as interrupções, três vezes por semana ${ }^{6,24,25}$, utilizando calçado adequado em terreno plano e velocidade moderada com sensação subjetiva de esforço através da Escala de Borg modificada entregue ao paciente entre 4 e 5 - pouco intenso a intenso. Para registro da caminhada era entregue ao paciente um diário de registro para anotação do dia, tempo de caminhada, quantas vezes parou para cessação da dor, distância e sensação de esforço e de dor (de 0 a 10) a fim de acompanhar a realização e evolução do exercício. O outro exercício orientado foi a flexão plantar, realizada em três séries, sendo que o número de repetições orientado foi 80\% do número máximo de repetições do HRT, três vezes por semana antes da caminhada ou em dias alternados. Nas avaliações mensais, o número de repetições a ser realizada era ajustado conforme o resultado do HRT. Os participantes foram orientados a evitar a realização de qualquer exercício extenuante no dia anterior e no dia das avaliações.

O estudo foi provado pelo Comitê de Ética da Universidade Federal de Minas Gerais sob o número 49801715.8.0000.5149.

Os dados foram apresentados como média e desviopadrão. A análise da normalidade dos dados foi avaliada pelo teste de Shapiro-Wilk. As comparações nos períodos propostos foram realizadas pela ANOVA de medidas repetidas com post-hoc least significant difference (LSD). Foi considerado para significância $p<0,05$.

\section{RESULTADOS}

Foram analisados 13 prontuários, sendo que dois não foram incluídos devido à ausência de registro da primeira avaliação de um mês de exercício não supervisionado. Sendo 
assim, foram incluídos no estudo 11 indivíduos na avaliação de um mês de atividade e 10 na análise de ambas avaliações.

A Tabela 1 apresenta os dados antropométricos expressos como média e desvio padrão (DP) ou frequências absoluta e relativa da amostra incluída no estudo.

Os valores de média e desvio padrão das variáveis do HRT (repetições e taxa de repetição), do ISWT (distância e economia de caminhada) e do questionário WIQ (diagnóstico diferencial, distância de caminhada, velocidade de caminhada e escadas) na avaliação inicial, no primeiro mês e no segundo mês de atividade física não supervisionada, além dos valores de $\mathrm{F}$ e $p$ obtidos por meio da análise de variância com medidas repetidas estão expressos na Tabela 2. Podemos observar uma melhora estatisticamente significativa no primeiro mês de atividade física não supervisionada em comparação à avaliação inicial, no que diz respeito ao número de repetições e na taxa de repetição no HRT, na distância de caminhada, na economia de caminhada no ISWT, e, no questionário WIQ há uma percepção de melhora na graduação da dor e velocidade de caminhada em indivíduos com DAP que realizaram o treinamento não supervisionado em casa através de orientações domiciliares mensais.

Analisando o segundo mês de atividade física, os indivíduos melhoram em relação à avaliação inicial nas mesmas variáveis que o primeiro mês, além de melhorarem a percepção da distância de caminhada no WIQ. Não foi verificada diferença no desempenho e na percepção da

Tabela 1. Características antropométricas da amostra do estudo $(n=11)$.

\begin{tabular}{lc}
\hline Variáveis & Média \pm DP \\
Idade (anos) & $64,09 \pm 8,51$ \\
Sexo masculino n (\%) & $7(63,64)$ \\
Altura (metros) & $1,60 \pm 0,77$ \\
Peso (quilogramas) & $73,88 \pm 17,38$ \\
IMC (quilograma/metro ${ }^{2}$ ) & $28,29 \pm 4,85$ \\
\hline
\end{tabular}

DP: Desvio padrão; IMC: Índice de massa corporal; n: Número.

Tabela 2. Resultados relativos ao HRT, ISWT e WIQ.

\begin{tabular}{|c|c|c|c|c|c|}
\hline Variáveis & Avaliação & 10 mês & 2o mês & $\mathbf{F}$ & $p$ \\
\hline Repetições HRT & $36,73 \pm 13,89$ & $49,91 \pm 21,88^{*}$ & $55,60 \pm 21,84^{\#}$ & 14,49 & 0,0001 \\
\hline Tempo HRT (segundos) & $55,27 \pm 19,01$ & $62,64 \pm 31,71$ & $68,60 \pm 22,92$ & 2,99 & 0,076 \\
\hline Taxa de repetição (repetições/segundo) & $0,68 \pm 017$ & $0,84 \pm 020^{*}$ & $0,82 \pm 018^{\#}$ & 7,73 & 0,004 \\
\hline Distância ISWT (metros) & $207,27 \pm 97,68$ & $266,36 \pm 113,87^{*}$ & $265 \pm 111,88^{\#}$ & 14,99 & 0,0001 \\
\hline Economia de caminhada (m/FC) & $2,07 \pm 1,02$ & $2,59 \pm 1,08^{*}$ & $2,56 \pm 1,08^{\#}$ & 11,10 & 0,001 \\
\hline Diagnóstico diferencial WIQ (\%) & $15,91 \% \pm 12,61$ & $43,18 \% \pm 25,23 *$ & $60 \% \pm 12,91^{\#}$ & 18,57 & 0,0001 \\
\hline Distância WIQ (\%) & $23,35 \pm 38,52$ & $33,02 \pm 34,53$ & $53,82 \pm 36,30^{\#}$ & 4,97 & 0,021 \\
\hline Velocidade WIQ (\%) & $21,84 \pm 26,91$ & $38,59 \pm 27,49^{*}$ & $47,22 \pm 23,47^{\#}$ & 5,33 & 0,017 \\
\hline Escada WIQ (\%) & $37,12 \pm 26,31$ & $49,58 \pm 35,54$ & $54,58 \pm 17,29$ & 1,45 & 0,26 \\
\hline
\end{tabular}

HRT: Heel-rise test; ISWT: Incremental Shuttle Walking Test; WIQ: Walking Impairment Questionnaire; m: Metros; FC: Frequência cadíaca.

* $p<0,051$ mês versus avaliação. * $p<0,052$ o mês versus avaliação. comparados o primeiro e o segundo mês de atividade física.

\section{DISCUSSÃO}

O presente estudo demonstrou que a atividade física não supervisionada apresentou resultados positivos na capacidade funcional, resistência muscular e da autopercepção de funcionalidade em indivíduos com DAP que completaram os dois meses de atividade física em casa com acompanhamento mensal.

Embora a evidência do benefício da atividade física não supervisionada seja limitada ${ }^{26}$ e apesar do programa supervisionado resultar em benefícios superiores em relação à capacidade de caminhada ${ }^{27,6}$, um programa altamente estruturado baseado no domicílio proporciona uma melhora funcional e pode ser uma alternativa satisfatória, gerando menos custos para os serviços de saúde ${ }^{27-29}$.

Os resultados obtidos nesse estudo foram satisfatórios e corroboram com os resultados encontrados na literatura, onde os programas de exercícios realizados em casa, quando apresentaram alta adesão, foram eficientes para melhorar o tempo de caminhada e a autopercepção de funcionalidade $\mathrm{e}^{5,26}$.

O estudo de Gardner et al.$^{30}$ mostrou que a adesão ao exercício domiciliar foi alta, cerca de $80 \%$, e aumentou o tempo de início da claudicação (204 137 segundos préteste e $337 \pm 250$ segundos pós-teste, $p<0,001$ ), tempo de caminhada máxima ( $402 \pm 285$ segundos pré-teste e $526 \pm 374$ pós-teste, $p<0,01$ ) e a percepção de funcionalidade no WIQ nos domínios distância (pré-teste $32 \pm 29 \%$ e $42 \pm 33 \%$ pósteste, $p<0,01$ ), velocidade (pré-teste $30 \pm 22 \%$ e $41 \pm 22 \%$ pós teste, $p<0,01$ ) e escadas (pré-teste $38 \pm 26 \%$ e $48 \pm 27 \%$ pós-teste, $p<0,01)^{30}$. Aqueles que completaram o programa de exercícios não supervisionado no estudo de Mouser et al. ${ }^{26}$, demonstraram uma melhora de $86,4 \%(p=0,001)$ na distância de para claudicação inicial e uma melhora de $19,8 \%(p=0,006)$ na distância até a claudicação máxima ${ }^{26}$. 
No presente estudo, apenas três pacientes não completaram todas as etapas de avaliação, correspondendo a 76,92\% de adesão. Em relação à capacidade funcional avaliada pela ISWT e a autopercepção de funcionalidade no WIQ, este estudo demonstrou resultados positivos.

Alguns estudos que avaliaram o resultado do treinamento não supervisionado não apresentavam um programa estruturado de orientação e avaliações programadas. Bendermacher et al. ${ }^{27}$, apresentou 14 estudos em uma revisão envolvendo pacientes com DAP que realizaram treinamento de exercícios supervisionados e não supervisionados. Segundo este estudo, os pacientes que realizaram exercícios supervisionados obtiveram resultados superiores na distância máxima com um tamanho de efeito global de 0,69 (95\% intervalo de confiança (I.C.), 0,51 para 0,86 ) e 0,48 (95\% I.C., 0,32 para 0,64), em seis e três meses de exercício, respectivamente. Os programas não supervisionados apresentaram variabilidade no modo de orientação domiciliar, alguns orientavam somente "ir para casa e caminhar" e outros apresentavam um programa de treinamento estruturado com orientações planejadas ${ }^{27}$.

A economia de caminhada é um dos mecanismos para melhorar as distâncias até a claudicação após o treinamento de exercícios. Com baixo custo de energia um paciente pode caminhar a uma determinada velocidade com menor esforço metabólico, facilitando a atividade e reduzindo os sintomas de claudicação ${ }^{19}$. O estudo de Gardner et al. ${ }^{30}$ relatou uma melhora na economia de caminhada com o treinamento supervisionado (pré-teste $10,06 \pm 2 \mathrm{~mL}^{\mathrm{kg}}{ }^{-1} \cdot \mathrm{min}^{-1}$ e pós-teste $\left.9,5 \pm 1,9 \mathrm{~mL} \cdot \mathrm{kg}^{-1} \cdot \mathrm{min}^{-1}, p<0,001\right)$, mas não mostrou melhora com o treinamento não supervisionado ${ }^{30}$. $\mathrm{O}$ aumento no desempenho do teste de caminhada mostrado neste estudo, somado a uma melhora na economia de caminhada após o treinamento representa uma melhora da capacidade metabólica induzida pelo exercício. No presente estudo, após um mês de atividade não supervisionada, foi verificada melhora do custo metabólico, representado pela economia de caminhada. Foi observado que os pacientes caminhavam maior distância sem ter que aumentar o consumo energético para exercer essa atividade, ou seja, conseguiam andar mais metros para cada batimento cardíaco.

O tratamento da DAP deve ser individualizado e com base em uma avaliação cuidadosa. A educação do paciente é primordial, tanto em relação as implicações da doença a longo prazo, quanto aos benefícios do exercício para esse tipo de paciente. $\mathrm{O}$ estabelecimento de terapêuticas adequadas de redução dos fatores de risco, a modificação de estilo de vida e terapias médicas somadas à prática de exercícios pode melhorar a condição clínica do paciente com DAP6 O programa domiciliar deve incluir também mecanismos que maximizem o retorno para acompanhamento, encorajamento a cada sessão, além de um feedback sobre os progressos e os resultados obtidos ${ }^{6,26}$.

Um estudo ${ }^{31}$ que teve como objetivo construir e validar um instrumento capaz de avaliar e descrever o conhecimento de pacientes com a finalidade de educação sobre sua doença, justifica a realização de tal instrumento, pois ajuda o paciente a obter uma percepção adequada de seu estado de saúde, possibilitando a modificação de crenças, comportamentos e hábitos ruins. O conhecimento sobre a condição de saúde é determinante para a aderência e o sucesso de seu tratamento ${ }^{32,33}$, sendo necessário um maior enfoque em uma educação sistematizada sobre a doença, seus fatores de risco e o tratamento realizado.

O presente estudo foi realizado com um programa de exercícios individualizados, predeterminados, com duração preestabelecida, orientações, monitoramento e feedback periódico, servindo como uma alternativa para melhorar os resultados em pacientes específicos, principalmente para aqueles com dificuldade de acesso aos serviços ambulatoriais. O volume de exercícios e a intensidade devem ser individualizados para cada paciente, além disso, os indivíduos devem ser orientados sobre os possíveis sinais e sintomas que indicam a necessidade de interrupção da atividade e sobre a importância do controle da intensidade do esforço por meio de escala de esforço.

A melhora no desempenho nos testes e na percepção da capacidade de caminhada observada no presente estudo foi detectada, principalmente, em algumas variáveis no primeiro mês de atividade física. Não foi verificada melhora no segundo mês comparado ao primeiro, demostrando uma estabilização dos ganhos obtidos no primeiro mês. Na maioria dos estudos, os programas de exercício consistiam em 12 semanas de treinamento e com acompanhamento longitudinal. O estudo de Patterson et al. ${ }^{28}$ demonstrou que um programa de exercícios semanal em casa com duração de 12 semanas, somados à palestras, resultou em melhora no aumento do tempo de claudicação máxima no grupo não supervisionado de 3,6 $6 \pm 2,73$ minutos para $6,6 \pm 3,17$ minutos após o seguimento de 6 meses. Para o grupo supervisionado o ganho foi maior (de $3,8 \pm 2,74$ inicial para 11,2 $\pm 4,02$ minutos após 6 meses). Resultados similares foram encontrados para o tempo máximo de caminhada ${ }^{28}$.

Gardner et al. ${ }^{34}$ mostraram em um estudo que os ganhos mediados pelo exercício ocorrem rapidamente nos primeiros 2 meses de reabilitação do exercício e são mantidos com treinamento adicional. Um programa de exercícios relativamente curto de 2 meses pode ser preferido para tratar pacientes com DAP, porque a adesão é maior, os 
custos associados ao pessoal e ao uso das instalações são mais baixos e mais pacientes podem ser treinados ${ }^{34}$.

No presente estudo, os indivíduos não observaram melhora na capacidade de subir e descer escadas, avaliada pelo domínio escadas do WIQ. Tal resultado pode ser justificado pelo fato de o único exercício de resistência realizado durante o estudo envolver apenas o músculo tríceps sural, o qual auxilia, mas não é o principal músculo envolvido no movimento de subir escadas. Um estudo que utilizou o treinamento de resistência mostrou melhora no tempo de caminhada e nas medidas de qualidade de vida, particularmente na capacidade de subir escadas, mostrando que o treino de resistência de membros inferiores, e não somente do músculo da panturrilha, melhora o desempenho em subir escadas em pacientes com DAP ${ }^{35}$.

Por se tratar de dados retrospectivos, o estudo apresenta limitações como a não inclusão de indivíduos que apresentavam nos prontuários informações faltantes, como por exemplo a não realização de no mínimo três avaliações mensais seguidas. Além disso, incluímos um numero reduzido de indivíduos com avaliações completas e não possuímos grupo controle, não podendo dessa forma, realizar conclusões amplas com os resultados obtidos. Como se trata de exercícios não supervisionados realizados em casa, os mesmos eram autorrelatados, mas para tentar controlar a realização ou não, os indivíduos deveriam anotar as informações necessárias no diário entregue no dia da avaliação inicial.

A partir do presente estudo foi possível concluir que o treinamento não supervisionado, realizado em casa, com orientações sistemáticas e individualizadas foi seguro e apresentou resultados satisfatórios nos domínios capacidade funcional, resistência muscular e autopercepção de funcionalidade para os indivíduos avaliados. Os resultados do presente estudo demonstram a importância da padronização de programas de exercícios, com acompanhamento sistematizado e encorajamento a cada sessão de forma detalhada e individualizada para treinamento de indivíduos com DAP.

\section{REFERÊNCIAS}

1. Hirsch AT, Haskal ZJ, Hertzer NR, Bakal CW, Creager MA, Halperin JL, Hiratzka LF, Olin JW, Puschett JB, Rosenfield KA, Sacks D, Stanley JC, TaylorJr LM, White CJ, White J, White RA. ACC/ AHA 2005 practice guidelines for the management of patients with peripheral arterial disease (lower extremity, renal, mesenteric, and abdominal aortic). Circulation. 2005;113(11):1474-547. https://doi.org/10.1161/circulationaha.106.174526

2. Vyskocil E, Gruther W, Steiner I, Schuhfried O. Identification of International Classification of Functioning, Disability and Health categories for patients with peripheral arterial disease. Am J Phys Med Rehabil. 2014;93(7):570-8. http://dx.doi.org/10.1097/ PHM.0000000000000063
3. Gardner AW, Katzel LI, Sorkin JD, Bradham DD, Hochberg MC, Flinn WR, Goldberg AP. Exercise rehabilitation improves functional outcomes and peripheral circulation in patients with intermittent claudication: a randomized controlled trial. J Am GeriatrSoc. 2001;49(6):755-62. http://dx.doi.org/10.1046/j. 1532-5415.2001.49152.x

4. Malgor RD, Alalahdab F, Elraiyah TA, Rizvi AZ, Lanem MA, Prokop LJ, Phung OJ, Farah W, Montori VM, Conte MS, Murad MH. A systematic review of treatment of intermittent claudication in the lower extremities. J Vasc Surg. 2015;61(3 Suppl):54S-73S. http://dx.doi.org/10.1016/j.jvs.2014.12.007

5. Gardner AW, Ritti-Dias RM, Stoner JA, Montgomery PS, Scott KJ, Blevins SM. Walking economy before and after the onset of claudication pain in patients with peripheral arterial disease. J Vasc Surg. 2011;51(3):628-33. http://dx.doi.org/10.1016/j. jvs.2009.09.053

6. Society for Vascular Surgery Lower Extremity Guidelines Writing Group, Conte MS, Pomposelli FB, Clair DG, Geraghty PJ, McKinsey JF, Mills JL, Moneta GL, Murad MH, Powell RJ, Reed AB, Schanzer A, Sidawy AN; Society for Vascular Surgery. Society for Vascular Surgery practice guidelines for atherosclerotic occlusive disease of the lower extremities: management of asymptomatic disease and claudication. J Vasc Surg. 2015;61(3 Supl):2S-41S. http://dx.doi.org/10.1016/j.jvs.2014.12.009

7. Patel MR, Conte MS, Cutlip DE, Dib N, Geraghty P, Gray W, Hiatt WR, Ho M, Ikeda K, Ikeno F, Jaff MR, Jones WS, Kawahara M, Lookstein RA, Mehran R, Misra S, Norgren L, Olin JW, Povsic TJ, Rosenfield K, Rundback J, Shamoun F, Tcheng J, Tsai TT, Suzuki Y, Vranckx P, Wiechmann BN, White CJ, Yokoi H, Krucoff MW. Evaluation and treatment of patients with lower extremity peripheral artery disease: Consensus definitions from peripheral academic research consortium (PARC). J Am Coll Cardiol. 2015;65(9):931-41. http://dx.doi.org/10.1016/j.jacc.2014. 12.036

8. Bermingham SL, Sparrow K, Mullis R, Fox M, Shearman C, Bradbury A, Michaels J. The cost-effectiveness of supervised exercise for the treatment of intermittent claudication. Eur J Vasc Endovasc Surg. 2013;46(6):707-14. http://dx.doi.org/10.1016/j. ejvs.2013.09.005

9. Al-Jundi W, Madbak K, Beard JD, Nawaz S, Tew GA. Systematic review of home-based exercise programmes for individuals with intermittent claudication. Eur J Vasc Endovasc Surg. 2013;46(6):690-706. http://dx.doi.org/10.1016/j.ejvs.2013. 09.004

10. Criqui MH, Langer RD, Fronek A, Feigelson HS, Klauber MR, McCann TJ, Browner D. Mortality over a period of 10 years in patients with peripheral arterial disease. N Engl J Med. 1992;326(6):381-6. http://dx.doi.org/10.1056/ NEJM199202063260605

11. Hamburg NM, Balady GJ. Exercise rehabilitation in peripheral artery disease: functional impact and mechanisms of benefits. Circulation. 2011;123(1):87-97. http://dx.doi.org/10.1161/ CIRCULATIONAHA.109.881888 
12. Locatelli EC, Pelizzari S, Scapini KB, Leguisamo CP, Silva AB. Exercícios físicos na doença arterial obstrutiva periférica. J Vasc Bras. 2009;8(3):247-54. http://dx.doi.org/10.1590/S167754492009000300010

13. Oliveira Fiho JA, Salvetti XM. Reabilitação não supervisionada ou semi-supervisionada: uma alternativa prática. Arq Bras Cardiol. 2004;83(5):368-70. http://dx.doi.org/10.1590/S0066$782 \times 2004001700002$

14. Olveira Filho JA, Leal AC, Lima VC, Santos Filho DV, Luna Filho B. Reabilitação não supervisionada: efeitos de treinamento ambulatorial a longo prazo. Arq Bras Cardiol. 2002;79(3):233-8. https://doi.org/10.1590/s0066-782×2004 001700002

15. Pereira DAG, Oliveira KL, Cruz JO, Souza CG, Cunha Filho IT. Avaliação da reprodutibilidade de testes funcionais na doença arterial periférica. Fisioter Pesq. 2008;15(3):228-34. http:// dx.doi.org/10.1590/S1809-29502008000300003

16. Monteiro DP, Britto RR, Lages AC, Basílio ML, Pires MCO, Carvalho ML, Procópio RJ, Pereira DA. Heel-rise test in the assessment of individuals with peripheral arterial occlusive disease. Vasc Health Risk Manag. 2013;9:29-35. http://dx.doi. org/10.2147/VHRM.S39860

17. Monteiro DP, Britto RR, Carvalho MLV, Montemezzo D, Parreira VF, Pereira DAG. Shuttle walking test como instrumento de avaliação da capacidade funcional: uma revisão da literatura. Rev Ciência Saúde. 2014;7(2):92-7. http://dx.doi.org/10.15448/ 1983-652X.2014.2.16580

18. Cunha-Filho IT, Pereira DA, Carvalho AM, Campedeli L, Soares M, Freitas JS. The reliability of walking tests in people with claudication. Am J Phys Med Rehabil. 2007;86(7):574-82. http://dx.doi.org/10.1097/PHM.0b013e31806de721

19. Womack CJ, Sieminski DJ, Katzel LI, Yataco A, Gardner AW. Improved walking economy in patients with peripheral arterial occlusive disease. Med Sci Sports Exerc. 1997;29(10): 1286-90. http://dx.doi.org/10.1097/00005768-19971000000002

20. Beneke R, Meyer K. Walking performance and economy in chronic heart failure patients pre and post exercise training. Eur J Appl Physiol Occup Physiol. 1997;75(3):246-51. http://dx.doi. org/10.1007/s004210050155

21. Ritti-Dias RM, Gobbo LA, Cucato GG, Wolosker N, Jacob Filho W, Santarém JM, Carvalho CRF, Forjaz CLM, Marucci MFN. Tradução e validação do Walking Impairment Questionnaire em brasileiros com claudicação intermitente. Arq Bras Cardiol. 2009;92(2):143-9. http://dx.doi.org/10.1590/S0066-782X2009 000200011

22. Sagar SP, Brown PM, Zelt DT, Pickett WL, Tranmer JE. Further clinical validation of the walking impairment questionnaire for classification of walking performance in patients with peripheral artery disease. Int J Vasc Med. 2012;2012:190641. http://dx.doi. org/10.1155/2012/190641
23. Myers SA, Johanning JM, Stergiou N, Lynch TG, Longo GM, Pipinos II. Claudication distances and the Walking Impairment Questionnaire best describe the ambulatory limitations in patients with symptomatic peripheral arterial disease. J Vasc Surg. 2008;47(3):550-5. http://dx.doi.org/10.1016/j.jvs.2007.10.052

24. Rooke TW, Hirsch AT, Misra S, Sidawy AN, Beckman JA, Findeiss L, Golzarian J, Gornik HL, Jaff MR, Moneta GL, Olin JW, Stanley JC, White CJ, White JV, Zierler RE; American College of Cardiology Foundation Task Force; American Heart Association Task Force. Management of patients with peripheral artery disease (compilation of 2005 and 2011 ACCF/AHA guideline recommendations): a report of the American College of Cardiology Foundation/American Heart Association Task Force on Practice Guidelines. J Am Coll Cardiol. 2013;61(14):1555-70. http://dx.doi.org/10.1016/j.jacc.2013.01.004

25. Lauret GJ, van Dalen DC, Willigendael EM, Hendriks EJ, de Bie RA, Spronk S, Teijink JA. Supervised exercise therapy for intermittent claudication: current status and future perspectives. Vascular. 2012;20(1):12-9. http://dx.doi.org/10.1258/vasc.2011. ra0052

26. Mouser MJ, Zlabek JA, Ford CL, Mathiason MA. Community trial of home-based exercise therapy for intermittent claudication. Vasc Med. 2009;14(2):103-7. http://dx.doi. org/10.1177/1358863X08098596

27. Bendermacher BL, Willigendael EM, Teijink JA, Prins MH. Supervised exercise therapy versus non-supervised exercise therapy for intermittent claudication. Cochrane Database Syst Rev. 2006;(2):CD005263. http://dx.doi.org/10.1002/14651858. CD005263.pub2

28. Patterson RB, Pinto B, Marcus B, Colucci A, Braun T, Roberts M. Value of a supervised exercise program for the therapy of arterial claudication. J Vasc Surg. 1997;25(2):312-9. http://dx.doi. org/10.1016/s0741-5214(97)70352-5

29. Galea MN, Weinman JA, White C, Bearne LM. Do behaviourchange techniques contribute to the effectiveness of exercise therapy in patients with intermittent claudication? A systematic review. Eur J Vasc Endovasc Surg. 2013;46(1):132-41. http:// dx.doi.org/10.1016/j.ejvs.2013.03.030

30. Gardner AW, Parker DE, Montgomery PS, Scott KJ, Blevins SM. Efficacy of quantified home-based exercise and supervised exercise in patients with intermittent claudication: a randomized controlled trial. Circulation. 2011;123(5):491-8. http://dx.doi. org/10.1161/CIRCULATIONAHA.110.963066

31. Ghisi GL, Durieux A, Manfroi WC, Herdy AH, Carvalho TD, Andrade A, Benetti M. Construction and validation of the CADE-Q for patient education in cardiac rehabilitation programs. Arq Bras Cardiol. 2010;94(6):813-22. http://dx.doi.org/10.1590/s0066$782 \times 2010005000045$

32. Jeon YH, Kraus SG, Jowsey T, Glasgow NJ. The experience of living with chronic heart failure: a narrative review of qualitative studies. BMC Health Serv Res. 2010;10:77. https://doi.org/10.1186/14726963-10-77 
33. Sommaruga M, Vidotto G, Bertolotti G, Pedretti RF, Tramarin R. A self administered tool for the evaluation of the efficacy of health educational interventions in cardiac patients. Monaldi Arch Chest Dis. 2003;60(1):7-15.

34. Gardner AW, Montgomery PS, Parker DE. Optimal exercise program length for patients with claudication. J Vasc Surg. 2012; 55(5):1346-54. http://dx.doi.org/10.1016/j.jvs.2011.11.123

35. McDermott MM, Ades P, Guralnik JM, Dyer A, Ferrucci L, Liu K, Nelson M, Lloyde-Jones, Van Horn L, Garside D, Kibbe M, Domanchuk K, Stein JH, Liao Y, Tao H, Green D, Pearce WH, Schneider JR, McPherson D, Laing ST, McCarthy WJ, Shroff A, Criqui MH. Treadmill exercise and resistance training in patients with peripheral arterial disease with and without intermittent claudication: a randomized controlled trial. Jama. 2009;301(2):165-74. http://dx.doi.org/10.1001/jama.2008.962

\section{Autoras:}

Carolina Machado de Melo Felix

Fisioterapeuta. Residente no Hospital das Clínicas da Universidade Federal de Minas Gerais (UFMG), Belo Horizonte, MG, Brasil.

Orcid: http://orcid.org/0000-0003-4236-3187

E-mail: carolmmfelix91@gmail.com

DÉBORA PANTUSO MONTEIRO

Fisioterapeuta. Doutora em Ciências da Reabilitação pela UFMG. Fisioterapeuta do Departamento de Fisioterapia da Universidade Federal de Minas Gerais (UFMG), Belo Horizonte, MG, Brasil.

Orcid: http://orcid.org/0000-0002-8531-7773

E-mail: deborapantuso@hotmail.com

DANielle APARECIDA GOMEs Pereira

Fisioterapeuta. Doutora em Ciências da Reabilitação pela UFMG. Professora do

Departamento de Fisioterapia e do Programa de Pós-Graduação em Ciências da

Reabilitação da Universidade Federal de Minas Gerais (UFMG), Belo Horizonte, MG

Brasil.

Orcid: http://orcid.org/0000-0003-4400-2326

E-mail: danielleufmg@gmail.com 